\title{
Channel direction information probing for multi-antenna cognitive radio system
}

\author{
Fang Yuan*, Gabriel Porto Villardi, Fumihide Kojima and Hiroyuki Yano
}

\begin{abstract}
This work studies the problem of channel direction information (CDI) probing for multi-antenna cognitive radio system. The CDI of the channel from the secondary transmitter (ST) to primary receiver (PR) is elementary information in designing the beamforming at the ST for mitigating the interference to the PR. However, lacking the explicit cooperation between primary and secondary systems, the CDI has to be acquired by probing at the ST, which is challenging. To solve this, we consider the line of sight (LOS) channel between the ST and the PR, and propose one CDI probing scheme for the ST. Specifically, the ST sends two types of probing signals by beamforming towards an interested region where both the secondary receiver (SR) and the PR are located and then actively learns the hidden feedback information from the primary system to acquire the CDI. The proposed scheme has a closed-form solution, and avoids the iteration between the probing and acquisition, which is desirable for practical system. Moreover, we show that the proposed probing scheme can be extended for primary systems working under multi-access channel and broadcasting channel. Simulation results demonstrate that the proposed scheme can improve the accuracy of the acquired CDI at the ST in cognitive ratio system remarkably.
\end{abstract}

\section{Introduction}

Cognitive radio (CR) is known as one of the promising techniques to meet the ever-increasing demand on spectrum efficiency in the future wireless systems[1]. It has attracted much attention in both the academic and the industry over the last decade[2-6].

Depending on the spectrum-sharing sharing strategies, there are generally two operation modes in the CR system. One is overlay mode, where the secondary transmission is enabled only when there is no primary transmission [1]. The other is underlay mode, where the secondary transmission is enabled if the interference to primary system can be tolerated $[2,4]$. Compared to the overlay mode, the underlay mode has more potential in improving the spectrum efficiency. Thus this work studies the underlay $\mathrm{CR}$ system.

To protect the primary system, one commonly used method is imposing an interference temperature constraint on the secondary transmitter (ST), such that the interference to the primary receiver (PR) is restricted to below a prescribed threshold. Various schemes have been

\footnotetext{
*Correspondence: yuanfang@nict.go.jp

Smart Wireless Laboratory, National Institute of Information and Communications Technology, Japan
}

proposed to improve the secondary transmission under the imposed constraint [7]. To implement these schemes, the channel information from the ST to the PR is required at the ST. However, such channel information is usually not available at the ST due to the lack of cooperation between primary and secondary systems [4].

To reduce the cooperation requirement between primary and secondary systems, several channel probing schemes [7-10] have been proposed for the ST to acquire the channel gain information from the ST to the PR (CGIsp). In [8], the ST is viewed as a relay and forwards the known useful signals to the PR in the probing. This scheme avoids the interference jamming to the PR led by the probing, but requires the ST to have the instantaneous data information sent from the primary transmitter (PT) to the PR. In [7], a framework is proposed for probing the CGIsp by exploiting the hidden feedback, e.g, the link adaptation information in primary system. In the scheme, the ST sends probing signals to interfere with the PR and learns the link adaptation information in primary transmission, through which the ST acquires the CGIsp. As indicated by [7], exploiting the hidden feedback information can reduce the cooperation requirement between primary and secondary systems significantly.

\section{黑 Springer}

(c) 2015 Yuan et al. Open Access This article is distributed under the terms of the Creative Commons Attribution 4.0 International License (http://creativecommons.org/licenses/by/4.0/), which permits unrestricted use, distribution, and reproduction in any medium, provided you give appropriate credit to the original author(s) and the source, provide a link to the Creative Commons license, and indicate if changes were made. 
It is desirable to apply multi-antenna techniques in the CR system, since the interference to the PR can be suppressed greatly by using the beamforming at the ST [11]. However, a proper beamforming requires the channel direction information from the ST to the PR (CDIsp) to be available at the ST. Similar to the CGIsp, the CDIsp has to be probed as well. However, the problem of CDIsp probing is more challenging than that of CGIsp probing. This is because the CDIsp is not so explicit in the hidden feedback information as the CGIsp. For example, the channel gain associated with the CGIsp can be measured directly with the strength of link adaptation in the hidden feedback information [7], while the channel direction associated with the CDIsp can not. The work in $[9,12]$ has proposed the algorithms to learn the null space information of the CDIsp from the hidden feedback information in primary system, which however, rely on the binary-search method. The binary-search method requires to adapt the probing signals with the received hidden feedback information until the acquired information is converged, which may cause too much overhead because of the iteration between the probing and acquisition. Therefore, it is more desirable to find a CDIsp probing scheme free of iteration for the multi-antenna CR system.

In this work, we consider for the ST to probe the CDIsp from the hidden feedback information in primary system under the line-of-sight (LoS) channel between the ST and the PR. The contributions of this work are twofold:

1. The CDIsp probing scheme is proposed for the multi-antenna CR system without requiring the explicit cooperation between primary and secondary systems. Specifically, the ST sends two types of probing signals to interfere with the PR by specially designed beamforming. Then, the ST learns the hidden feedback information in primary transmission and acquire the target CDIsp. The proposed CDIsp probing scheme has a closed-form solution and is free of iteration between the probing and acquisition, which is desirable in CR system.

2. The proposed CDIsp probing scheme is extended for primary systems under multi-access channel (MAC) and broadcasting channel (BC). We demonstrate that for primary system under MAC channel, the proposed scheme can be applied with incremental power detection techniques, and for primary system under BC channel, the proposed scheme can be applied with parametric estimation techniques. The results show that the proposed scheme works very well under practical conditions.

The rest of this work is organized as follows. We first present the system model in Section 2. In Section 3, we propose the basic CDIsp probing scheme under the singleuser CR system. Then in Section 4, the proposed scheme is extended for primary systems under MAC and BC channels, respectively. Simulation results are provided in Section 5, and the work is concluded in Section 6.

\section{System model}

\subsection{The general model of the hidden feedback information}

Consider an underlay CR system where the primary and secondary systems are deployed, respectively, in the micro and small cells. Figure 1 shows an example for the basic single-user CR system. The ST with multiple antennas mitigates the undesirable interference to the PR by beamforming, for which the CDI of the channel from the ST to the PR (i.e., CDIsp) needs to be probed at the ST.

Let $P_{t}$ be the transmit power at the PT, and $\rho$ be the target signal to interference-and-noise ratio (SINR) at the $\mathrm{PR}$ in primary transmission. It satisfies that

$$
\frac{P_{t} \lambda_{u}}{\sigma^{2}+I}=\rho
$$

where $\sigma^{2}$ is the additive receiver-noise power at the PR, and $I$ is the interference at the PR from the ST. More system parameters about the basic $\mathrm{CR}$ system are listed in Table 1.

The secondary system can perform active learning on the hidden feedback information from the primary system in the probing [4]. The hidden feedback information is the link adaptation information in primary system, e.g., data rate, transmit power or others, which can be observed and detected by the ST without a dedicated channel to perform the explicit cooperation between the primary and secondary systems. In this work, the hidden feedback information is considered as the adaptation of transmit power at the PT, where the primary system has a constant data rate with a target SINR of $\rho$ at the PR. However, it should be noted that the proposed scheme can be easily extended for other types of hidden feedback information in [4].

During the primary transmission, the ST receives the signal power from the PT as

$$
P_{f}=P_{t} \lambda_{f}=\frac{\rho \lambda_{f}}{\lambda_{u}}\left(\sigma^{2}+I\right)
$$

where the last equality is from (1). When the ST does no probing, i.e., $I=0$, the received signal power at the ST becomes

$$
P_{f, 0}=\frac{\rho \lambda_{f}}{\lambda_{u}} \sigma^{2} .
$$

By differentiating the received signal powers with and without the probing, the ST acquires the power adaptation information from primary system as

$$
\Xi=P_{f}-P_{f, 0}=\frac{\rho \lambda_{f}}{\lambda_{u}} I,
$$




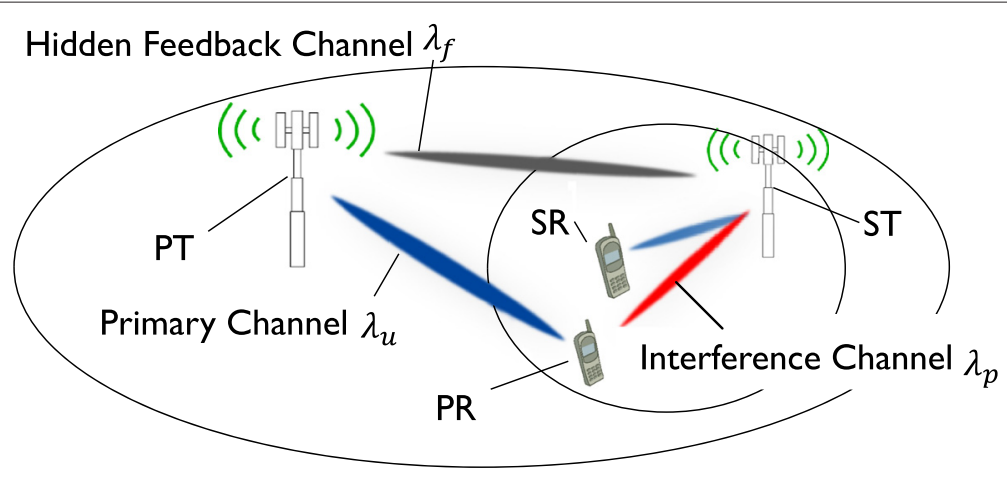

(a)Basic CR System

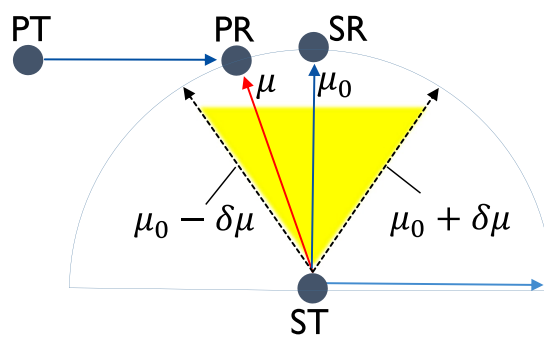

(b)Location in CR system

Fig. 1 An example of the multi-antenna CR system

Table 1 Key system parameters in the basic CR system

\begin{tabular}{ll}
\hline$\lambda_{u}$ & the channel gain from the PT to the PR \\
$\lambda_{f}$ & the channel gain from the PT to the ST \\
$\lambda_{p}$ & the channel gain from the ST to the PR \\
$\mu_{0}$ & the angle position of SR \\
$\mu / \hat{\mu}$ & the angle/acquired angle position of PR \\
$h / \bar{h}(\mu)$ & the channel vector/the CDI from the PT to the ST \\
$N$ & the number of antennas at the ST \\
$\delta \mu$ & the half-range of probing region \\
$P_{t} / P_{0}$ & the transmit power at the PT/ST \\
$P_{f} / P_{f, 0}$ & the received signal power at the ST from the PT under \\
$\rho$ & probing/no probing \\
$\sigma^{2}$ & the target SINR at the PR \\
$g$ & the receiver noise power at the PR \\
$l_{1} / I_{2}$ & the probing gain at the ST \\
$\Xi_{1} / \Xi_{2}$ & $\begin{array}{l}\text { the interference at the PR led by the 1th/2nd type of } \\
\text { probing signal } \\
\text { the hidden feedback information from the PT to the ST led } \\
\text { by the 1th/2nd type of probing signal }\end{array}$ \\
& the ratio for the CDlsp with the PR
\end{tabular}

which is a monotonic function of the interference led by the probing. The value of $\Xi$ can be observed at the ST without the need of a dedicated channel between the PT and ST, and therefore is regarded as the hidden feedback information from the primary system to the secondary system.

As explained in [4], the quality of the acquired hidden feedback information in (4) may be degraded by several imperfections in the probing, e.g., the receiver noise or unexpected interference, the measurement errors at the ST. These imperfections have been carefully discussed in [4] for the probing of CGIsp, and the conclusions show that the probing performance is robust to small imperfections. In general, these imperfections are not unique to the problem studied here, but widely observed to exist in many other probing schemes for the CR system $[3,8,9,12]$. Therefore, to concentrate on the idea of the CDIsp probing itself, we leave these imperfections fuzzy in the design, and assume that the acquired hidden feedback information is perfect at the ST in Section 3 and 4. The impact of the imperfections on the CDIsp probing performance is evaluated comprehensively via the simulations in Section 5.

\subsection{LoS channel model and the interested probing region} Consider that the antennas are closely placed at the ST into a uniform linear array (ULA), which is common in 
practical systems due to the limited form factor. According to the LoS channel model in $[13,14]$, the channel from the ST to the PR, is expressed as [15]

$$
\boldsymbol{h}=a\left[1, e^{-j \mu}, \cdots, e^{-j(N-1) \mu}\right]^{T},
$$

with $\mu=2 \pi \frac{d}{v} \cos \phi$, where $a$ is the instantaneous complex channel gain, $d$ and $N$ are, respectively, the antenna spacing and the number of antennas at the ST, $v$ is the carrier wavelength, $\phi$ is the physical angle of the PR's location respect to the broadside of ST, $j^{2}=-1$ and ()$^{T}$ means the transpose operation. We consider that the channel is subject to the block fading, which is regarded as constant during the probing.

It is significant to study the LoS channel in wireless communications. The LoS channels are usually observed in outdoor open areas under lower frequency bands [13]. As the carrier frequency expands into milli-meter wave bands in the future systems, LoS channels can be very typical due to less reflections, and are considered more beneficial for narrow beamforming in interference mitigation $[16,17]$.

The CDIsp is defined as

$$
\overline{\boldsymbol{h}}(\mu)=\left[1, e^{-j \mu}, \cdots, e^{-j(N-1) \mu}\right]^{T},
$$

which is of constant-norm and only conveys the channel direction information in $\boldsymbol{h}$ [15]. Under LoS channel, acquiring the CDIsp is equivalent to acquiring the angle value $\mu$. With the CDIsp of $\overline{\boldsymbol{h}}(\mu)$ at the ST, a proper beamforming can be designed for mitigating the interference to the PR [11], e.g., the pseudo-inverse zero-forcing (ZF) beamforming vector in [15].

Denote the ST sends the probing signal $s$ by using the beamforming vector $f$ towards the PR in the probing. The received instantaneous interference signal at the $\mathrm{PR}$ is

$$
y_{\text {ins }}=\boldsymbol{h}^{H} \boldsymbol{f} \text {, }
$$

where ()$^{H}$ is the Hermitian operation. From (7), the interference in (1) and (4) is further written as

$$
I=\left|y_{\text {ins }}\right|^{2}=P_{0} \lambda_{p}\left|\overline{\boldsymbol{h}}(\mu)^{H} \boldsymbol{f}\right|^{2}
$$

where || is the absolute value, $P_{0}=|s|^{2}$ is the probing power at the ST, $\lambda_{p}=|a|^{2}$ is the channel gain in $\boldsymbol{h}$.

Using (8), the hidden feedback information in (4) at the ST from primary system is expressed by

$$
\Xi=g\left|\overline{\boldsymbol{h}}(\mu)^{H} \boldsymbol{f}\right|^{2},
$$

where $g=\frac{P_{0} \rho \lambda_{f} \lambda_{p}}{\lambda_{u}}$ is the probing gain at the ST.

Considering that the beamwidth at the ST is limited, instead of full angle region, the ST is usually interested to probe for only a specific region around the secondary receiver (SR), where the PR may be severely interfered without the CDIsp at the ST. Figure 1(b) illustrates the location relationship in the CR system, where the PR and the SR are located, respectively, at the angle $\mu$ and $\mu_{0}$ from the view of the ST.

Denote the angle region interested for probing around the $\mathrm{SR}$ as

$$
\left[\mu_{0}-\delta \mu, \mu_{0}+\delta \mu\right]
$$

where $\delta \mu$ is the half of the range of probing region. The setting of $\delta \mu$ depends on the uncertainty about the angle position of PR at the ST. A large value of $\delta \mu$ covers a larger probing region suitable for a larger uncertainty about the PR, and vice versa. For simplicity, we set $\delta \mu$ as multiple times of the null-beamwidth of the antenna array at the ST, i.e.,

$$
\delta \mu=\frac{2 \ell \pi}{N}
$$

where $\ell=1, \ldots, \frac{N}{4}$ is an integer such that $\delta \mu \leq \frac{\pi}{2}$.

By setting $\ell,(11)$ allows for a flexibility to cover the needs of different probing range up to $\pi$. In this work, we assume the probing range is pre-given in the probing. The impact of $\delta \mu$ on the probing performance is evaluated in the simulation section.

\section{The basic CDIsp probing scheme}

We first consider the basic CDIsp probing scheme for the single-user CR system as shown in Fig. 1. The idea of the probing scheme is to design the interference in (8) by chosing a specific beamforming vector $f$ during the probing, such that the different hidden feedback information from primary system conveys different CDIsp.

To do this, we propose that the ST probes with the two beamforming vectors targeted, respectively, at the direction of $\mu_{0}+\delta \mu$ and $\mu_{0}-\delta \mu$ as

$$
\begin{aligned}
& \boldsymbol{f}_{1}(\delta \mu)=\frac{1}{\sqrt{N}}\left[1, e^{-j\left(\mu_{0}+\delta \mu\right)}, \cdots, e^{-j(N-1)\left(\mu_{0}+\delta \mu\right)}\right]^{T} \\
& \boldsymbol{f}_{2}(\delta \mu)=\frac{1}{\sqrt{N}}\left[1, e^{-j\left(\mu_{0}-\delta \mu\right)}, \cdots, e^{-j(N-1)\left(\mu_{0}-\delta \mu\right)}\right]^{T} .
\end{aligned}
$$

By sending the probing signal $s$ with $f=f_{1}(\delta \mu)$ into the primary system, the hidden feedback information in (9) for the first type of probing signal becomes

$$
\begin{aligned}
\Xi_{1} & =g\left|\overline{\boldsymbol{h}}(\mu)^{H} \boldsymbol{f}_{1}(\delta \mu)\right|^{2} \\
& =\frac{g}{N} \frac{\sin ^{2} \frac{N\left(\mu-\mu_{0}-\delta \mu\right)}{2}}{\sin ^{2} \frac{\left(\mu-\mu_{0}-\delta \mu\right)}{2}} \\
& =\frac{g}{N} \frac{\sin ^{2} \frac{N\left(\mu-\mu_{0}\right)}{2}}{\sin ^{2} \frac{\left(\mu-\mu_{0}-\delta \mu\right)}{2}},
\end{aligned}
$$

where (14) is from the equality $\left|\sum_{n=1}^{N} e^{-j x}\right|^{2}=\frac{\sin ^{2} \frac{N x}{2}}{\sin ^{2} \frac{x}{2}}$, and (15) is by using (11). 
Similarly, with $\boldsymbol{f}=\boldsymbol{f}_{2}(\delta \mu)$, the hidden feedback information in (9) for the second type of probing signal becomes

$$
\begin{aligned}
\Xi_{2} & =g\left|\overline{\boldsymbol{h}}(\mu)^{H} f_{2}(\delta \mu)\right|^{2}=\frac{g}{N} \frac{\sin ^{2} \frac{N\left(\mu-\mu_{0}+\delta \mu\right)}{2}}{\sin ^{2} \frac{\left(\mu-\mu_{0}+\delta \mu\right)}{2}} \\
& =\frac{\lambda}{N} \frac{\sin ^{2} \frac{N\left(\mu-\mu_{0}\right)}{2}}{\sin ^{2} \frac{\left(\mu-\mu_{0}+\delta \mu\right)}{2}} .
\end{aligned}
$$

Then we can find a ratio via the differentiation and the sum on two hidden feedback information at the ST as

$$
r=\frac{\Xi_{1}-\Xi_{2}}{\Xi_{1}+\Xi_{2}}=\frac{-\sin \left(\mu-\mu_{0}\right) \sin \delta \mu}{1-\cos \left(\mu-\mu_{0}\right) \cos \delta \mu}
$$

which conveys the CDIsp and is irrespective of the channel gains.

For the PR within the interested probing range, i.e., $\left|\mu-\mu_{0}\right| \leq \delta \mu$, it is easy to show that $r$ is a monotonously decreasing function with $\mu-\mu_{0}$. For example, the value of $r$ as a function of $\mu-\mu_{0}$ with $\delta \mu=\pi / 2, \pi / 4, \pi / 8$ and $N=16$ are given in Fig. 2. By using the ratio from the hidden feedback information, the ST can immediately acquire the value of $\mu$ in the CDIsp through the reverse function as

$\hat{\mu}=\mu_{0}-\arcsin \left(\frac{r \sin \delta \mu-r \sqrt{1-r^{2}} \sin \delta \mu \cos \delta \mu}{\sin ^{2} \delta \mu+r^{2} \cos ^{2} \delta \mu}\right)$.

With the acquired $\hat{\mu}$, the CDIsp can be reconstructed as $\overline{\boldsymbol{h}}(\hat{\mu})$, which is identical to $\overline{\boldsymbol{h}}(\mu)$ in (6) if the ratio $r$ is perfect such that $\hat{\mu}=\mu$.
Only a few scalar computations are needed in the probing, e.g., for the learning of the hidden feedback information in (15) and (16), and the closed-form solution in (17) and (18). Therefore, the computational complexity in the proposed scheme is low. The probing scheme does not require the iteration between the probing and acquisition, and thus the probing overhead is reduced when compared to the iteration-based probing schemes [9]. Moreover, the proposed probing can be applied under any channel gain and any target SINR, since the value of $r$ is irrespective of the probing power, the channel gain, and the target SINR.

As mentioned in the previous section, the quality of the hidden feedback information may be degraded by the imperfections in the probing. To increase the robustness for practical system, it can start from a large probing range intended to capture a coarse CDIsp and end in a small probing range to acquire a fine CDIsp. In this case, both the setting of probing center $\mu_{0}$ and half-probing range $\delta \mu$ are gradually adapted and refined to reduce the impact of the imperfections.

\section{Extensions for primary systems under MAC and BC channel}

In this section, we extend the probing scheme for primary systems working under $\mathrm{MAC}$ and $\mathrm{BC}$ channel respectively, which is more realistic than the single-user $C R$ system.

We assume that the interested probing region has a number of PRs known by the ST, and the impact from outside of the probing region can be ignored. The

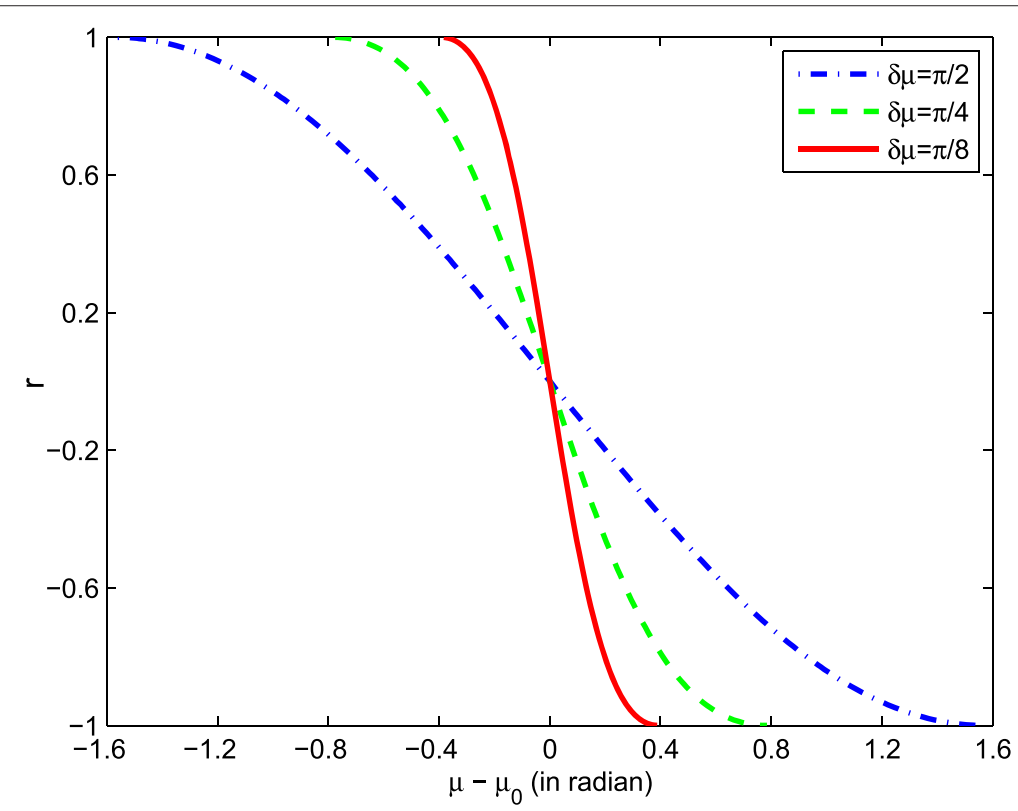

Fig. 2 The value of the ratio $r$ as a function of $\mu-\mu_{0}$ under $N=16$ 
number of PRs can be obtained at the ST by using the multi-target identification techniques [18], which solve the typical estimation problem of how many unknown targets exist in the system [19]. The multitarget identification can be performed before the CDIsp probing.

For simplicity, the index $i$ is used to refer the different type of probing signal. Specifically, $i=0,1,2$ means no probing signal, the first type of probing signal by using (12) and the second type of probing signal by using (13), respectively.

\subsection{Primary systems under MAC channel}

As illustrated in Fig. 3a, when the PR works as an access point with multiple PTs connected to it in uplink, the ST may interfere with the PR, and thus the CDIsp with the angle $\mu$ in the channel from the ST to the PR needs to be probed. Here, the hidden feedback channels are the ones from all the PTs to the ST. Still, there is no explicit cooperation between the PTs and the ST.

Without loss of generality, we consider that there are two PTs in MAC channel, i.e., PT A and PT B, and the probing scheme can be easily extended to the case of more than two PTs.

Incremental power detection at the ST: When the primary system works under MAC channel, the ST receives signals from both PT A and PT B. Let $s_{k}$ be the instantaneous data symbol intended to the PR from the PT $k$, and $P_{f, k}$ be the corresponding received signal power of $s_{k}$ at

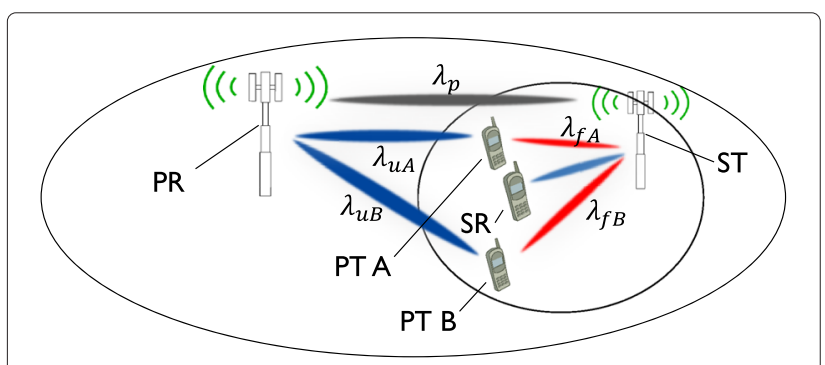

(a)Primary System under MAC Channel

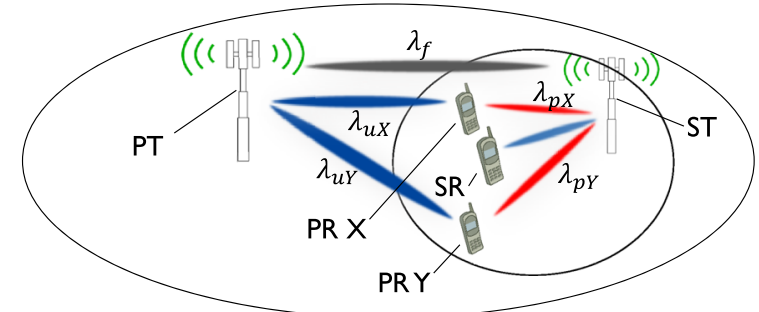

(b)Primary System under BC Channel

Fig. 3 Primary systems under MAC and BC channel the ST, where $k=A, B$. Then, the $\mathrm{ST}$ receives a sum of transmit signals from the two PTs as

$$
y_{\text {mac }}=\sum_{k \in\{A, B\}} \sqrt{P_{f, k}} s_{k} .
$$

Assuming the $s_{A}$ and $s_{B}$ are independently identically distributed (i.i.d.), the total received signal power at the ST is expressed as

$$
E\left\{\left|y_{\text {mac }}\right|^{2}\right\}=\sum_{k \in\{A, B\}} P_{f, k},
$$

where $E\{\}$ is the expectation operation. In practice, the total received signal power in (20) can be detected at the ST by using the second moment of the measured samples $y_{\text {mac }}$.

Let $y_{i, m a c}$ be the sample of $y_{\text {mac }}$ measured at the ST during no probing signal $(i=0)$, the first type of probing signal $(i=1)$ and the second type of probing signal $(i=2)$. Then the total incremental power of the received signal at the ST led by the first and second type of probing signal is expressed as

$$
\begin{aligned}
& \Xi_{1, \text { mac }}=E\left\{\left|y_{1, \text { mac }}\right|^{2}\right\}-E\left\{\left|y_{0, \text { mac }}\right|^{2}\right\}, \\
& \Xi_{2, \text { mac }}=E\left\{\left|y_{2, \text { mac }}\right|^{2}\right\}-E\left\{\left|y_{0, \text { mac }}\right|^{2}\right\},
\end{aligned}
$$

which are further used in the CDIsp probing under MAC channel.

Probing under MAC channel: Denote the transmit power at the PT $k$ as $P_{t, i, k}$ under the $i$ th type of probing signal. The target SINR $\rho_{k}$ at the PR due to the PT $k$ is given as

$$
\frac{P_{t, i, k} \lambda_{u, k}}{\sigma^{2}+I_{i, k}}=\rho_{k}
$$

where $i=1,2$ and $k=A, B$. Additionally, the interference $I_{i, k}$ at the PR due to the PT $k$ led by the $i$ th type of probing signal is expressed as

$$
I_{i, k}=P_{0} \lambda_{p}\left|\overline{\boldsymbol{h}}^{H}(\mu) \boldsymbol{f}_{i}(\delta \mu)\right|^{2} .
$$

More system parameters with MAC channel are listed in Table 2.

As in (4), the individual received incremental signal power at the ST from the PT $k$ due to the $i$ th type of probing signal can be derived as

$$
\Xi_{i, k}=\frac{\rho_{k} \lambda_{f, k}}{\lambda_{u, k}} I_{i, k}=g_{k}\left|\overline{\boldsymbol{h}}^{-H}(\mu) \boldsymbol{f}_{i}(\delta \mu)\right|^{2},
$$

where $g_{k}=\frac{P_{0} \rho_{k} \lambda_{f, k} \lambda_{p}}{\lambda_{u, k}}$ is the probing gain via the PT $k$.

The hidden feedback information under MAC channel is the total incremental power at the ST received from both PT A and PT B, which is expressed by

$$
\Xi_{i, m a c}=\sum_{k \in\{A, B\}} \Xi_{i, k} .
$$


Table 2 Additional system parameters with MAC channel

\begin{tabular}{ll}
\hline$k \in\{A, B\}$ & PT A or B under MAC channel \\
\hline$\lambda_{u, k}$ & the channel gain from the PT $k$ to the PR \\
$\lambda_{f, k}$ & the channel gain from the PT $k$ to the ST \\
$y_{\text {mac }}$ & the received signal at the ST \\
$y_{i, m a c}$ & the received signal at the ST under the ith type of probing \\
& signal \\
$P_{f, k}$ & the received signal power at the ST from the PT $k$ \\
$P_{t, i, k}$ & the transmit power at the PT $k$ under the ith type of probing \\
& signal \\
$\rho_{k}$ & the target SINR at the PR from the PT $k$ \\
$l_{i, k}$ & the interference at the PR for the PT $k$ led by the ST under \\
& the ith type of probing signal \\
$g_{k}$ & the probing gain via the PT $k$ \\
$\Xi_{i, m a c}$ & the hidden feedback information to the ST under the ith \\
& type of probing signal
\end{tabular}

The hidden feedback information in (26) can be acquired at the ST by incremental power detection as given in (21) and (22).

From (25) and (26), we can find that

$$
\begin{aligned}
r & =\frac{\Xi_{1, m a c}-\Xi_{2, m a c}}{\Xi_{1, m a c}+\Xi_{2, m a c}} \\
& =\frac{\left(\sum_{k \in\{A, B\}} g_{k}\right)\left(\left|\overline{\boldsymbol{h}}^{H}(\mu) \boldsymbol{f}_{1}(\delta \mu)\right|^{2}-\left|\overline{\boldsymbol{h}}^{H}(\mu) \boldsymbol{f}_{2}(\delta \mu)\right|^{2}\right)}{\left(\sum_{k \in\{A, B\}} g_{k}\right)\left(\left|\overline{\boldsymbol{h}}^{H}(\mu) \boldsymbol{f}_{1}(\delta \mu)\right|^{2}+\left|\overline{\boldsymbol{h}}^{H}(\mu) \boldsymbol{f}_{2}(\delta \mu)\right|^{2}\right)} \\
& =\frac{-\sin \left(\mu-\mu_{0}\right) \sin \delta \mu}{1-\cos \left(\mu-\mu_{0}\right) \cos \delta \mu}
\end{aligned}
$$

which is similar to (17) except that the probing gain $\sum_{k \in\{A, B\}} g_{k}$ becomes a sum of the probing gain via each PT. Thus, the ST can use the hidden feedback information $\Xi_{1, \text { mac }}$ and $\Xi_{2, \text { mac }}$ from the two types of probing signals to find the value of $r$ for acquiring the CDIsp as in (18).

As as result, the proposed CDIsp probing scheme can be extended for primary system working under MAC channel by using the incremental power detection techniques. Compared to under the single-user CR system, the computation complexity under MAC channel is trivial, since (20) to (22) use only regular expectation operations.

\subsection{Primary systems under BC channel}

As illustrated in Fig. 3b, when the PT works as an access point connecting multiple PRs in downlink, the ST may interfere with multiple PRs, and thus all the CDIsp from each PR to the ST needs to be probed.

Without loss of generality, we assume that there are two PRs in downlink under BC channel, i.e., PR X and
PR Y, and two CDIsp with the angle $\mu_{X}$ and $\mu_{Y}$. Moreover, the pre-processing at the PT, e.g., ZF beamforming, is performed for downlink such that the two primary transmissions do not interfere with each other. Extensions can be easily made for more than two PRs. The problem of probing multiple CDIsp under BC channel is more complicated than that of probing one CDIsp under MAC channel. In the following, we show that it is still possible to apply the proposed probing scheme if parametric estimation techniques are considered.

To probe each CDIsp, the ST has to learn the individual transmit power adaptation for each PR in primary system. However, the ST receives a sum of transmit signals from the PT for two PRs under the $\mathrm{BC}$ channel, rather than two individual transmit signals. Therefore, the ST has to estimate the individual signal power for each PR from the received sum signals.

Parameter estimation at the ST: The problem of estimating individual received signal power at the ST can be modeled as follows. Let $s_{m}$ be the instantaneous data symbol intended to the PR $m$ from the PT, and $P_{f, m}$ be the corresponding received signal power at the $\mathrm{ST}$, where $m=X, Y$. Then the $\mathrm{ST}$ receives a sum of transmit signals from two PTs as

$$
y_{b c}=\sum_{m \in\{X, Y\}} \sqrt{P_{f, m}} s_{m} .
$$

The samples of $y_{b c}$ can be measured at the ST directly, but the parameters $P_{f, m}$ are not known to the ST and needed to be estimated. The problem of estimating the parameter $P_{f, m}$ from the samples of $y_{b c}$ is known as "parametric estimation", which is typical in the estimation theory [19]. There are many classical solutions, such as maximum likelihood and the moment method. In the following, we briefly introduce the the moment method to show the feasibility for the CDIsp probing when primary system works under BC channel.

As indicated in [19], the moment method is to estimate the unknown parameters of random variables from different-order moments of measured samples. For simplicity, we assume the random data information $s_{m}$ is i.i.d. and zero mean. Denote the second and fourth moments of $s_{m}$, respectively, as

$$
E\left\{\left|s_{m}\right|^{2}\right\}=\alpha_{m}, \text { and } E\left\{\left|s_{m}\right|^{4}\right\}=\beta_{m}
$$

The value of $\alpha_{m}$ and $\beta_{m}$ can be viewed as the prior knowledge at the ST, since the modulation scheme of $s_{m}$ in (28) is finite and can be blindly detected at the ST [20].

From (28) and (29), the moments of the received signal at the ST are

$$
\begin{aligned}
& E\left\{\left|y_{b c}\right|^{2}\right\}=P_{f, X}^{2} \alpha_{X}+P_{f, Y}^{2} \alpha_{Y} \\
& E\left\{\left|y_{b c}\right|^{4}\right\}=P_{f, X}^{4} \beta_{X}+P_{f, Y}^{4} \beta_{Y}+6 P_{f, X} P_{f, Y} \alpha_{X} \alpha_{Y} .
\end{aligned}
$$


The value of $E\left\{\left|y_{b c}\right|^{2}\right\}$ and $E\left\{\left|y_{b c}\right|^{4}\right\}$ can be obtained from the measured samples by the ST. By catenating (30) and (31), we can immediately find the solutions for $P_{f, X}$ and $P_{f, Y}$. In case of multiple solutions, higher-order moments can be catenated with (30) and (31) to resolve the unique solution.

Therefore, the individual received signal power for each PR can be estimated by the ST when using the parametric techniques. Let $P_{f, i, m}$ be the estimated received signal power at the ST intended to the PR $m$ during no probing signal $(i=0)$, the first type of probing signal $(i=1)$, and the second type of probing signal $(i=2)$. Then the individual incremental received power at the ST is expressed as

$$
\begin{aligned}
& \Xi_{1, m}=P_{f, 1, m}-P_{f, 0, m}, \\
& \Xi_{2, m}=P_{f, 2, m}-P_{f, 0, m},
\end{aligned}
$$

which are further used in the probing under $\mathrm{BC}$ channel.

Probing under BC channel: Under BC channel, the PT transmits power to the PR $m$ satisfying

$$
\frac{P_{t, i, m} \lambda_{u, m}}{\sigma^{2}+I_{i, m}}=\rho_{m},
$$

where $i=1,2, m \in\{X, Y\}$, and $I_{i, m}$ is the interference at the PR $m$ led by the $i$ th type of probing signal given as

$$
I_{i, m}=P_{0} \lambda_{p, m}\left|\overline{\boldsymbol{h}}^{H}\left(\mu_{m}\right) \boldsymbol{f}_{i}(\delta \mu)\right|^{2},
$$

\begin{tabular}{|c|c|}
\hline$m \in\{X, Y\}$ & PT X or Y under BC channel \\
\hline$\lambda_{p, m}$ & the channel gain from the ST to the PR $m$ \\
\hline$\lambda_{u, m}$ & the channel gain from the PT to the PR $m$ \\
\hline$\mu_{m}$ & the angle position of the PR $m$ \\
\hline$\rho_{m}$ & the target SINR at the PR $m$ \\
\hline$y_{b c}$ & the received signal at the $S T$ \\
\hline$P_{f, m}$ & the received signal power at the ST for the PR $m$ \\
\hline$P_{t, i, m}$ & $\begin{array}{l}\text { the transmit power to the PR } m \text { under the ith type of } \\
\text { probing signal }\end{array}$ \\
\hline$P_{f, i, m}$ & $\begin{array}{l}\text { the received power at the ST for the PR } m \text { under the } i \text { th } \\
\text { type of probing signal }\end{array}$ \\
\hline$l_{i, m}$ & $\begin{array}{l}\text { the interference at the PR } m \text { led by the ST under the ith } \\
\text { type probing signal }\end{array}$ \\
\hline$\Xi_{i, m}$ & $\begin{array}{l}\text { the hidden feedback information to the ST for the PR } m \\
\text { under the ith type of probing signal }\end{array}$ \\
\hline$g_{m}$ & the probing gain via the PR $m$ \\
\hline$r_{m}$ & the ratio for the CDIsp with the PR $m$ as in (17) \\
\hline
\end{tabular}

More system parameters with $\mathrm{BC}$ channel are listed in Table 3.

Table 3 Additional system parameters with BC channel
As given by (32) and (33), the hidden feedback information of individual power adaptation can be acquired at the ST. Similar to (4), the received incremental signal power at the ST from the PT $m$ due to the $i$ th type of probing signal can be derived as

$$
\Xi_{i, m}=P_{f, i, m}-P_{f, 0, m}=\frac{\rho_{m} \lambda_{f, m}}{\lambda_{u, m}} I_{i, m}=g_{m}\left|\overline{\boldsymbol{h}}^{H}\left(\mu_{m}\right) \boldsymbol{f}_{i}(\delta \mu)\right|^{2},
$$

where $g_{m}=\frac{P_{0} \rho_{m} \lambda_{p, m} \lambda_{f}}{\lambda_{u m}}$ is the probing gain via the PR $m$.

The CDIsp with the PR $m$ is conveyed by the ratio with the individual hidden feedback information as

$$
r_{m}=\frac{\Xi_{1, m}-\Xi_{2, m}}{\Xi_{1, m}+\Xi_{2, m}}=\frac{-\sin \left(\mu_{m}-\mu_{0}\right) \sin \delta \mu}{1-\cos \left(\mu_{m}-\mu_{0}\right) \cos \delta \mu},
$$

Then by using (18), the ST is able to obtain the CDIsp $\overline{\boldsymbol{h}}\left(\hat{\mu}_{X}\right)$ and $\overline{\boldsymbol{h}}\left(\hat{\mu}_{Y}\right)$.

As a result, the proposed CDIsp probing scheme is extended for primary systems working under $\mathrm{BC}$ channel by using parametric estimation techniques. Compared to under MAC channel, the CDIsp probing for primary system under BC channel may have a slightly higher computational complexity led by the parametric estimation. This is because the more PRs under $\mathrm{BC}$ channel, the more high-order moments are needed to catenate with (30) and (31) in order to estimate more received signal powers at the ST. In practice, considering the equations for higherorder moments are non-linear in general, the existing numerical methods are available to reduce the computation complexity efficiently, e.g., the Newton-Raphson method for solving non-linear equations [21].

\section{Simulation results}

The performance of the proposed CDIsp probing scheme is investigated via simulations for $\mathrm{CR}$ systems in this section.

\subsection{Impact of imperfections on the probing performance} This subsection investigates the probing performance under the imperfections, such as the measurement errors and the Non-LoS component in the channel. The basic single-user CR system shown in Fig. 1 is considered.

To model different levels of measurement errors, we assume the hidden feedback information in (9) at the ST is corrupted by an additive noise as

$$
\Xi=g\left|\overline{\boldsymbol{h}}(\mu)^{H} \boldsymbol{f}\right|^{2}+n,
$$

where $n$ is Gaussian distributed. The additive noise can be led by many practical factors, such as the receiver noise, unexpected interference, or low sensitivity at the ST. Define the measurement signal to noise ratio (SNR) as $g / E\left\{|n|^{2}\right\}$. The smaller the measurement SNR is, the lower quality of hidden feedback information the ST can 
measure. Therefore, the level of measurement errors are characterized by the measurement SNRs.

Figure 4 provides the mean-square error (MSE) of $\hat{\mu}$, i.e., $E\left\{|\hat{\mu}-\mu|^{2}\right\}$, versus different measurement SNR, where the number of antennas at the ST is $N=16$ and two values of $\delta \mu$ for the probing range are set. The results for two exemplary locations of PR are provided, where the angle separations between the PR and the SR are set as $\mu-\mu_{0}=\pi / 9$, and $\mu-\mu_{0}=\pi / 12$ respectively. As shown in Fig.4, the MSE of $\hat{\mu}$ is slightly different with the location of PR. This is because the value of $\left|\overline{\boldsymbol{h}}(\mu)^{H} \boldsymbol{f}\right|^{2}$ in the measured hidden feedback information $\Xi$ is different with the locations of PR. However, as the measurement SNR increases, the MSE of $\hat{\mu}$ is reduced in spite of the PR's locations. When $\delta \mu=\pi / 8$, the MSE of $\hat{\mu}$ for both locations is dropped to a low level, e.g., $10^{-2}$, even at a measurement SNR of $5 \mathrm{~dB}$. The results indicate that the proposed CDIsp probing scheme works very well at the moderate measurement errors.

To further consider the imperfections led by the NonLoS component in the channel, we assume the channel between the ST and the PR is modeled as the Rician channel, i.e.,

$$
\boldsymbol{h}=\sqrt{\frac{K}{1+K}} a \overline{\boldsymbol{h}}(\mu)+\sqrt{\frac{K \lambda_{p}}{1+K}} \boldsymbol{q},
$$

where $K$ is the Rician factor, and $\boldsymbol{q}$ is the Non-LoS component in the channel with i.i.d. complex Gaussian entries of zero mean and unit variance. The smaller the Rician factor is, the stronger the Non-LoS component in the channel becomes. Therefore, the strength of Non-LoS component is characterized by the value of $K$.

Figure 5 provides the MSE of $\hat{\mu}$ versus different Rician factor $K$, where $N=16$ and $\delta \mu=\pi / 8$. The results are provided under the measurement SNR of $5 \mathrm{~dB}$ and $15 \mathrm{~dB}$, respectively, and for two exemplary locations of PR $\mu-\mu_{0}=\pi / 9$ and $\mu-\mu_{0}=\pi / 12$. As shown in Fig. 5, the MSE of $\hat{\mu}$ is reduced when the Rician factor increases. When the Rician factor is about $10 \mathrm{~dB}$, the MSE of $\hat{\mu}$ is dropped to the level $10^{-2}$ approximately for both locations at the measurement SNR of $5 \mathrm{~dB}$. As the Rician factor becomes larger, the MSE of $\hat{\mu}$ cannot be reduced, and there is a floor effect on the MSE. This is because in the large $K$ regime, the MSE is mainly dominant by the measurement SNR, and increasing the measurement SNR is more effective to improve the MSE of $\hat{\mu}$.

\subsection{Probing when primary system works under BC and MAC channel}

This subsection considers more realistic cases where the primary system works under BC and MAC channel, as shown in Fig. 3.

We assume that quadrature amplitude modulation (QAM) is employed in primary transmission, i.e., $s_{k}$ in (19) and $s_{m}$ in (28) are QAM symbols. The number of antennas at the $\mathrm{ST}$ is $N=16$ and the Rician factor in the probing channel is set as $15 \mathrm{~dB}$. Without loss of generality, the target SINR at the PR and the probing power at the ST are set as one. To avoid the setting of individual channels in the CR system, we consider that all the channel gains $\lambda_{c}$ ( $c=p, f, u$ and etc.) in Fig. 3 are assumed to be $\lambda_{s n r}$, and

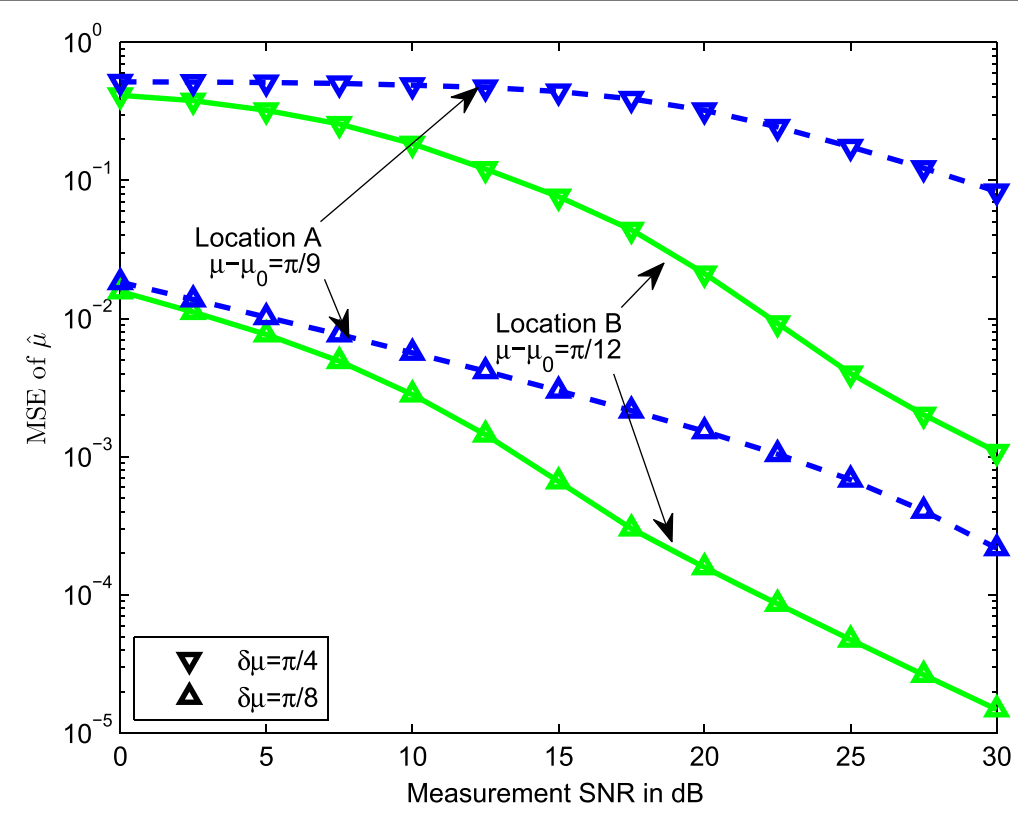

Fig. 4 Impact of measurement error on the basic CR system, where $N=16$ 


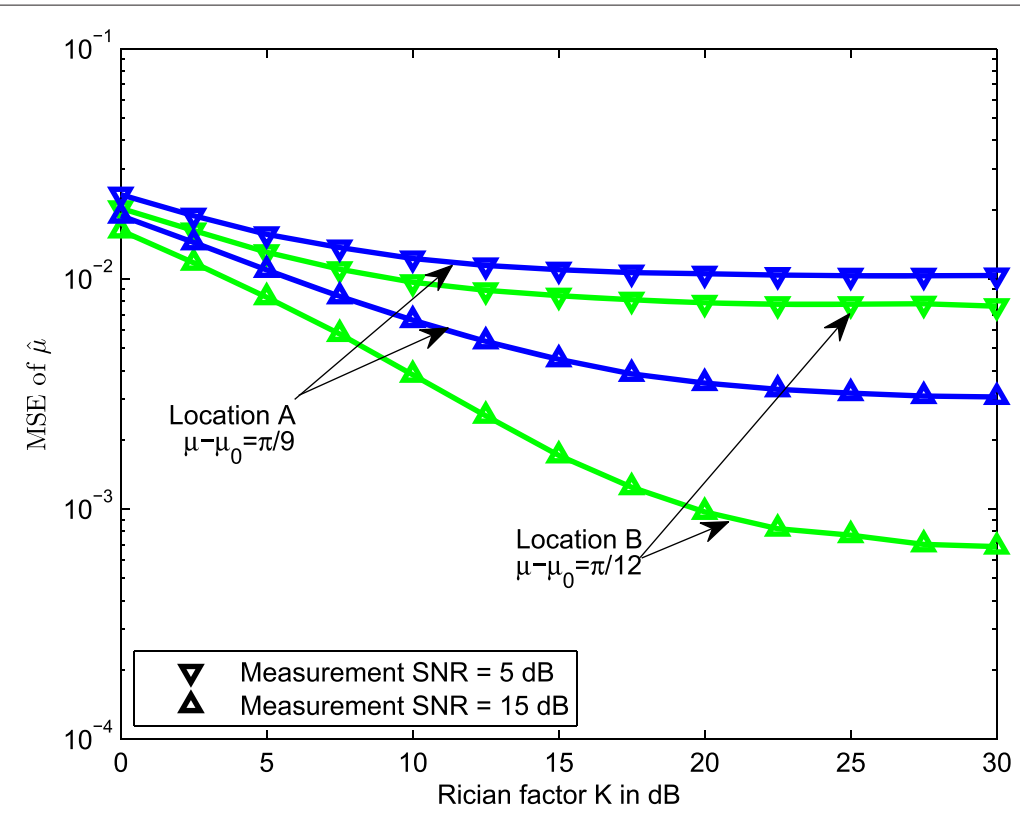

Fig. 5 Impact of Rician channel on the basic CR system, where $N=16$ and $\delta \mu=\pi / 8$

the receiver noise powers are set to one. This means the SNR of each channel is set equally as $\lambda_{s n r}$. Similar conclusions can be drawn from simulation results for CR systems with unequal setting parameters, which are not provided due to the limited space.

The received signal at the ST in (19) and (28) is corrupted by the receiver noise, and the impact of receiver noise is reflected by the channel SNR $\lambda_{s n r}$. Denote $N_{s}$ as the number of samples $y_{m a c}$ in (19) and $y_{b c}$ in (28) measured by the ST under each type of probing signal. Specifically, the moments are calculated as

$$
E\left\{|y|^{2 n}\right\} \approx \frac{1}{N_{s}} \sum_{\iota=1}^{N_{s}}\left|y^{(\iota)}\right|^{2 n},
$$

where $y^{(\iota)}$ is the $\iota$ th measurement of $y_{\text {mac }}$ under $n=1$ or $y_{b c}$ under $n=1,2$.

Under MAC channel: Fig. 6 investigates the impact of the PR's location on the CDIsp probing performance when the primary system has two PTs and one PR under MAC channel. The primary transmission for both two PRs are set as 16-QAM, and $N_{s}=8$ is set for (40).

As shown in the Fig. 6, the MSE of $\hat{\mu}$ varies according to the PR's location. The peaks of the MSEs happen to the PRs located at a separation angle of $\left|\mu-\mu_{0}\right|=$ $2 \pi / N \approx 0.4$ or $\left|\mu-\mu_{0}\right|=0$ from the SR. In the case of $\left|\mu-\mu_{0}\right|=2 \pi / N$, the reason for a large MSE is that either $\left|\overline{\boldsymbol{h}}^{H}(\mu) \boldsymbol{f}_{1}(\delta \mu)\right|^{2}$ or $\left|\overline{\boldsymbol{h}}^{H}(\mu) \boldsymbol{f}_{2}(\delta \mu)\right|^{2}$ in (25) is zero, such that the corresponding power adaptation in (26) is more easily corrupted by the receiver noise. In the case of $\left|\mu-\mu_{0}\right|=0$, the reason is that $\left|\overline{\boldsymbol{h}}^{H}(\mu) \boldsymbol{f}_{1}(\delta \mu)\right|^{2}=$ $\left|\overline{\boldsymbol{h}}^{H}(\mu) \boldsymbol{f}_{2}(\delta \mu)\right|^{2}$, such that the ratio of (27) becomes zero and is more easily corrupted by the receiver noise. However, in spite of the PR's location, the MSE of $\hat{\mu}$ in the CR system can be reduced as the channel SNR and the number of measured samples increases.

Under BC channel: Fig. 7 and Fig. 8 provide the CDIsp probing performance when the primary system has one PT and two PRs under BC channel, where the modulation order is set as the same for two PTs. The moment method is applied to estimate the received signal power at the ST. To investigate the impact of imperfections on the moment method, three issues are mainly evaluated: the modulation order in primary transmission, the channel SNR, and the number of measured samples at the ST. In the setting, the first PT is fixed at the location $\mu-\mu_{0}=\pi / N$, and the second PT is uniformly distributed within $\left|\mu-\mu_{0}\right| \leq \delta \mu$. The MSE of the probed CDIsp is shown for the first PT, while similar results can be observed for the PT with the other locations.

Figure 7 provides the simulation results of the CDIsp probing performance under different QAM scheme in the primary transmission and different channel SNR in the CR system. As shown in the figure, as the channel SNR increases, the MSE of $\hat{\mu}$ decreases remarkably in spite of the modulation orders. For a given number of measured sample, the MSE is decreased when the modulation order in primary transmission is reduced from 64QAM to 4QAM. This is because compared to the higher-order QAM symbols, the lower-order QAM 


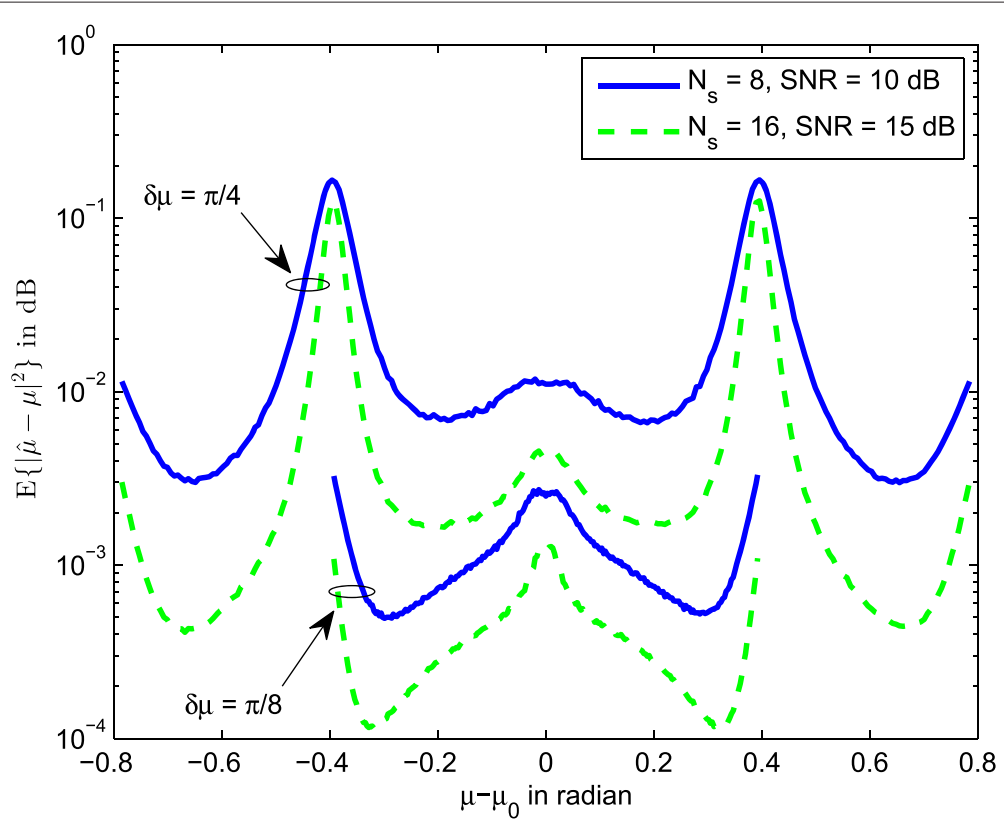

Fig. 6 Probing performance for primary system under MAC channel, where $N_{s}=8$

symbols have a less dynamic magnitude and the moments in (30) and (31) can be averaged out more accurately by using (40) with the same number of measured samples.

Figure 8 provides the simulation results of the CDIsp probing performance under different number of measured samples at the ST. As shown in the figure, as the number of measured samples increases, the MSE of $\hat{\mu}$ decreases remarkably under all the modulation orders for both the two probing range $\delta \mu=\pi / 4$ and $\delta \mu=\pi / 8$. To reduce the impact led by the QAM symbols, only a few measured samples, e.g., $N_{s}=10$ to 20 , are required to learn the hidden feedback information in the proposed CDIsp probing scheme.

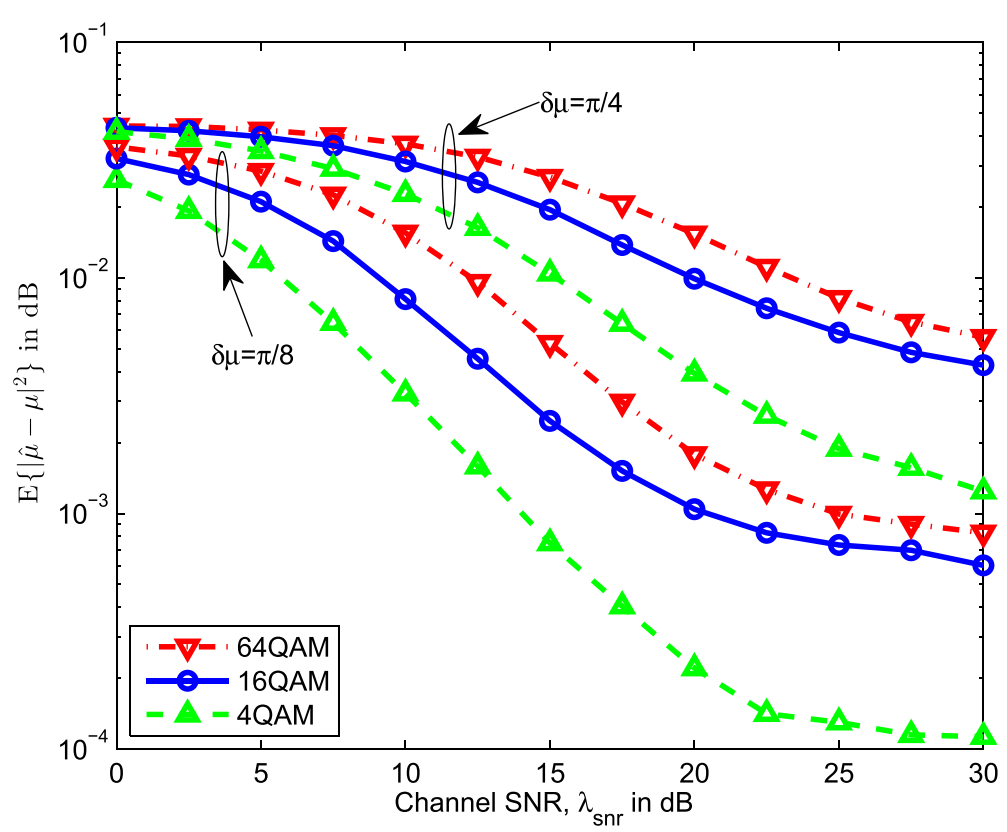

Fig. 7 Probing performance under BC channel with different channel SNR and QAM, where $N_{s}=20$ 


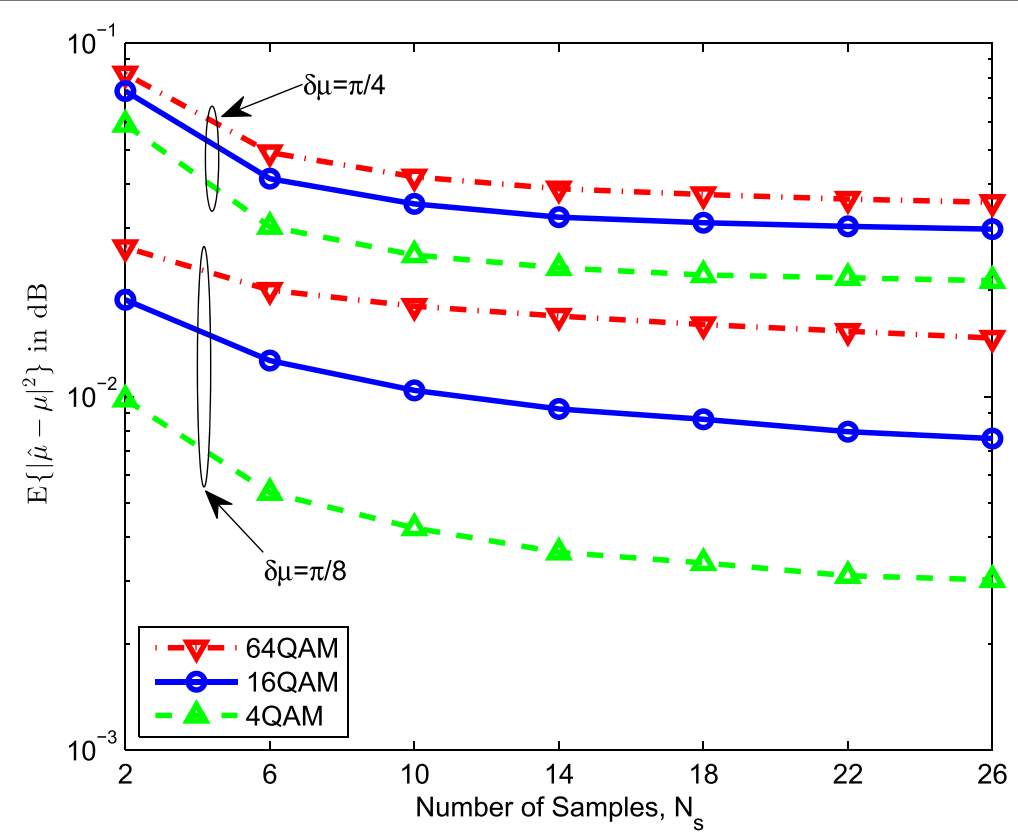

Fig. 8 Probing performance under $B C$ channel with different $N_{s}$ and $Q A M$, where $S N R=10 \mathrm{~dB}$

\section{Conclusions}

In this work, we have studied the problem of the CDIsp probing for CR system with multiple antennas under LoS channels. The CDIsp probing scheme by exploiting the hidden feedback in primary system has been proposed, with a solution free of iteration and of low complexity. The proposed scheme avoids the explicit cooperation between primary and secondary systems, and is irrespective of channel gains and target SINRs. Moreover, the proposed scheme has been extended for primary systems working under MAC channel and BC channel, respectively. Our simulation results have shown that the proposed scheme is efficient for the ST to acquire the CDIsp in CR system.

\section{Competing interests}

The authors declare that they have no competing interests.

\section{Acknowledgements}

This work is supported by the R\&D projects by Smart Wireless Laboratory, National Institute of Information and Communications Technology (NICT), Japan. The authors would like to thank the anonymous reviewers for their valuable comments and suggestions to improve the quality of the paper.

Received: 6 April 2015 Accepted: 8 August 2015

Published online: 27 August 2015

\section{References}

1. S Haykin, Cognitive radio brain-empowered wireless communications. IEEE J. Sel. Areas Commun. 23(2), 201-220 (2005)

2. A Jovicic, $P$ Viswanath, Cognitive radio: an information-theoretic perspective. IEEE Trans. Inf. Theory. 55(9), 3945-3958 (2011)

3. G Zhao, GY Li, C Yang, Proactive detection of spectrum opportunities in primary systems with power control. IEEE Trans. Wireless Commun. 8(9), 4815-4823 (2009)
4. R Zhang, On active learning and supervised transmission of spectrum sharing based cognitive radios by exploiting hidden primary radio feedback. IEEE Trans. Commun. 58(10), 2960-2970 (2010)

5. G Villardi, Y Alemseged, C Sun, C Sum, N Tran, T Baykas, H Harada, Enabling coexistence of multiple cognitive networks in tv white space. IEEE Wireless Communi. 18(4), 32-40 (2011)

6. G Zhao, C Yang, GY Li, D Li, ACK Soong, Power and channel allocation for cooperative relay in cognitive radio networks. IEEE J. Sel. in Signal Processing. 5(1), 151-159 (2011)

7. R Zhang, YC Liang, S Cui, Dynamic resource allocation in cognitive radio networks. IEEE Signal Process. Mag. 27(3), 102-114 (2010)

8. L Zhang, G Zhao, G Wu, Z Chen, in IEEE Global Telecommuni. Conf. (GLOBECOM). Proactive channel gain estimation for coexistence between cognitive and primary users (California, US, 2012)

9. Y Noam, A Goldsmith, Blind null-space learning for MIMO underlay cognitive radio with primary user interference adaptation. IEEE Trans. Wireless Commun. 12(4), 1722-1734 (2013)

10. G Zhao, W Shi, L Li, S Li, Passive primary receiver detection for underlay spectrum sharing in cognitive radio. IEEE Signal Processing Letters. 21(5), 564-568 (2014)

11. R Zhang, YC Liang, Exploiting multi-antennas for opportunistic spectrum sharing in cognitive radio networks. IEEE J. Sel. Topics Signal Process. 2(1), 88-102 (2008)

12. Y Noam, AG Goldsmith, in IEEE Int. Conf. Commun. (ICC). Blind null-space learning for spatial coexistence in MIMO cognitive radios (Ottawa,CA, 2012)

13. WINNER II Channel Models. D1.1.2 V1.1, online: www.ist-winner.org

14. D Tse, P Viswanath, Fundamentals of Wireless Communication. (Cambridge University Press, UK, 2005)

15. N Ravindran, N Jindal, HC Huang, in Proc. IEEE Global Telecommun. Conf. (GLOBECOM). Beamforming with finite rate feedback for LoS MIMO downlink channels (Washington, DC, US, 2007)

16. S Rangan, TS Rappaport, E Erkip, Millimeter-wave cellular wireless networks: Potentials and challenges. Proceedings of the IEEE. 102(3), 366-385 (2014)

17. DW Yue, GY Li, LoS-based conjugate beamforming and power-scaling law in massive-MIMO systems (2014). http://arxiv.org/pdf/1404.1654.pdf 
18. H-ST Le, Power control and multi-target identification in cognitive wireless networks. Master's thesis, Univ. of Texas at Arlington (2007)

19. HLV Trees, KL Bell, Z Tian, Detection Estimation and Modulation Theory, Part I, Detection, Estimation, and Filtering Theory, 2nd edn. (Wiley, US, 2013)

20. OA Dobre, A Abdi, Y Bar-Ness, W Su, Survey of automatic modulation classification techniques: classical approaches and new trends. IET Commun. 1(2), 137-156 (2007)

21. WH Press, SA Teukolsky, WT Vetterling, BP Flannery, Numerical Recipes: The Art of Scientific Computing, 3rd edn. (Cambridge Univ. Press, UK, 2007)

Submit your manuscript to a SpringerOpen ${ }^{\mathcal{O}}$ journal and benefit from:

- Convenient online submission

Rigorous peer review

- Immediate publication on acceptance

- Open access: articles freely available online

- High visibility within the field

- Retaining the copyright to your article

Submit your next manuscript at $\boldsymbol{\triangleright}$ springeropen.com 